\title{
Stable Susceptibility to Aminoglycosides in an Age of Low Level, Institutional Use
}

\author{
John A. Bosso • Martha L. Haines · Juanmanuel Gomez
}

To view enhanced content go to www.infectiousdiseases-open.com

Received: August 28, 2013 / Published online: October 22, 2013

(C) The Author(s) 2013. This article is published with open access at Springerlink.com

\section{ABSTRACT}

Introduction: The use of aminoglycosides has decreased dramatically over several decades in the United States due to the introduction of safer Gram-negative agents. This study was conducted to assess possibly changing aminoglycoside susceptibility rates between 2006 and 2012 and in reference to 1992 use in the context of aminoglycoside use volume.

Methods: Quarterly adult use of amikacin, gentamicin and tobramycin were determined from the Medical University of South Carolina Medical Center, Charleston, South Carolina, USA, pharmacy drug use database

J. A. Bosso $(\bowtie) \cdot$ M. L. Haines

South Carolina College of Pharmacy, Charleston, SC 29425, USA

e-mail: bossoja@musc.edu; bossoja@sccp.sc.edu

J. A. Bosso · J. Gomez

Medical University of South Carolina College of Medicine, Charleston, SC 29425, USA

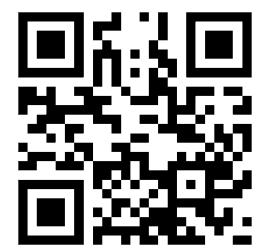

Enhanced content for this article is available on the journal web site: www.infectiousdiseases-open.com and expressed as total aminoglycoside defined daily doses per 1,000 patient days for the years 1992 and 2006 through 2012. Annual susceptibility of Pseudomonas aeruginosa, Escherichia coli, and Klebsiella pneumoniae, for the years 1992, 2006, and 2008 through 2012 were retrieved from our hospital's clinical microbiology database (duplicate isolates were excluded). Quarterly and annualized aminoglycoside usage rates were compared to the other years of interest. Likewise, susceptibility rates of the target organisms to each aminoglycoside were also compared across the same timeframe.

Results: While total use of aminoglycosides decreased slightly from 1992 to 2006, it increased by about 40\% between 2006 and 2008 and then stabilized. Changes in susceptibility rates between 1992 and 2006 were all $\leq \pm 9 \%$ with the exception of $K$. pneumoniae susceptibility to amikacin $(-17 \%)$. Changes in susceptibility from 1992 to 2012 were also all $\leq \pm 9 \%$. Tobramycin remained the most active versus $P$. aeruginosa (\% susceptible $=90)$, while amikacin remained most active versus E. coli and K. pneumoniae (\% susceptible $=98$ and 98 , respectively). 
Conclusion: With low level use of aminoglycosides in our institution over the past 2 decades, the susceptibility of key Gramnegative pathogens has remained relatively stable, preserving these agents as potential alternative therapies as resistance arises to other frequently used antibiotics.

Keywords: Amikacin; Aminoglycosides; Antibiotic usage; Antibiotic resistance; Escherichia coli; Gentamicin; Infectious diseases; Klebsiella pneumoniae; Pseudomonas aeruginosa; Tobramycin

\section{INTRODUCTION}

In the 1970s and 1980s, the aminoglycoside antibiotics were a key antibiotic group in the treatment of serious Gram-negative infections. With the introduction of new beta-lactam agents with pronounced Gram-negative activity during the 1980s, the use of aminoglycosides waned as the less toxic beta-lactams were increasingly used, and this trend continued into the early part of this century $[1,2]$. The declining use of one or more of the aminoglycosides was frequently accompanied by observations of increasing susceptibility among key pathogens $[3,4]$ although this relationship has not held true in all studies [2]. We are now entering a time in which we are encountering rapidly increasing Gram-negative resistance to broad-spectrum beta-lactams including third and fourth generation cephalosporins, beta-lactam-betalactamase inhibitor combinations, and the carbapenems. This rising resistance is often mediated by extended-spectrum betalactamases (ESBL) and carbapenemases [5-7]. Moreover, the Gram-negative pathogens producing these enzymes are often co-resistant to other important antibiotic classes such as the fluoroquinolones [7-9]. Because of this, it has been suggested by a number of studies that the use of aminoglycosides may be increasing as clinicians search for viable alternative therapies in treating infections with otherwise resistant Gram-negative pathogens [10-12]. The purpose of the present analysis was to assess the level of aminoglycoside use in adults at our institution from 2006 through 2012 and, during that same time period, the level of susceptibility of key Gram-negative pathogens to this antibiotic class.

\section{METHODS}

This study was conducted at the Medical University of South Carolina Medical Center, a 709-bed academic medical center located in Charleston, South Carolina, USA. The study was approved by the Medical University of South Carolina Medical Center Institutional Review Board. This article does not contain any studies with human or animal subjects performed by any of the authors. Pertinent data were assembled and analyzed for the period 2006 through 2012. Susceptibility data for the years 1992, 2006, and 2008 through 2012 for Pseudomonas aeruginosa, Escherichia coli (nonurine isolates only), and Klebsiella pneumoniae were obtained from the hospital's annual antibiograms which are produced in accordance with Clinical and Laboratory Standards Institute (CLSI) guidance [13]. Thus, no duplicate or surveillance isolates are included. Susceptibility was determined by an automated system (MicroScan WalkAway ${ }^{\circledR}$, Siemens Medical Solutions USA, Inc., Malvern, Pennsylvania, USA) in our Clinical Microbiology Laboratory throughout the period of interest for E. coli and K. pneumoniae although a change was made to Kirby-Bauer disk diffusion for $P$. aeruginosa in 2007 due to reported inaccuracies 
of automated systems in determining antibiotic susceptibility of this organism [14]. Systemic, adult usage data for amikacin, gentamicin and tobramycin for the years 1992 and 2006 through 2012 were obtained from the Department of Pharmacy Services drug administration records. Usage from these records is based on patient billing such that they account for doses dispensed but not returned to the pharmacy (or otherwise wasted) and therefore are, to the best of our knowledge, administered to the patients. Susceptibility data were expressed as percent susceptible and antibiotic usage data were transformed to defined daily doses (DDD) presuming the following typical adult doses: amikacin $15 \mathrm{mg} / \mathrm{kg} /$ day; gentamicin and tobramycin $7 \mathrm{mg} / \mathrm{kg} /$ day and assuming an $80 \mathrm{~kg}$ adult (DDDs $=1.2,0.56$ and $0.56 \mathrm{~g}$, respectively) which are more typical to dosing in this country (as opposed to those DDD definitions provided by the World Health Organization). Usage was normalized for hospital census [DDD/1,000 patient days (PD)]. In addition to these data for 2006 through 2012, data were also obtained for 1992 to provide a longer term perspective on potential changes in use and susceptibility. Although little change in total aminoglycoside use or susceptibility of the organisms of interest was noted in the last 4 years of analysis, 2012 values for each was compared to 1992 levels by Student's $t$ or Chisquared tests as appropriate using Excel $^{\circledR}$ for Mac 2011, version 14.3.7 (Microsoft Corporation, Washington, USA).

\section{RESULTS}

Results for antibiotic usage and organism susceptibility for the years of interest are presented in Tables 1 and 2, respectively. Simple visual inspection revealed little variation in susceptibility of the organisms of interest between 1992 and 2012 or in the last 4 years of observation and changes were not statistically significant. Figure 1 is illustrative of this observation, in this case for $P$. aeruginosa. Changes in susceptibility rates between 1992 and 2006 were all $\leq \pm 9 \%$ with the exception of $K$. pneumoniae susceptibility to amikacin (17\%). Changes in susceptibility from 1992 to 2012 were also all $\leq \pm 9 \%$. Tobramycin remained the most active versus $P$. aeruginosa (\% susceptible $=90$ ), while amikacin remained most active versus E. coli and K. pneumoniae (\% susceptible $=98$ and 98 , respectively). While total aminoglycoside use increased by almost $40 \%$ between 1992 and 2012, most of that increase occurred between 2006 and 2008 with only a $1 \%$ change in total DDD/1,000 PD between 1992 and 2006 and a 3\% increase occurring between 2008 and 2012, indicating stable levels of use during that final 5-year period. The mix of aminoglycosides used did change between 1992 and later years from a predominance of amikacin and gentamicin use to predominant tobramycin use.

\section{DISCUSSION}

In distinction to reports from other centers, we observed little change in the utilization of aminoglycosides in our institution in recent years (2008-2012) [1, 2]. Total aminoglycoside usage did increase almost $40 \%$ as compared to 1992 levels, however, and the make-up of total usage changed from amikacin predominance to tobramycin predominance over that time period. Nonetheless, as compared to use of other antibiotics for Gram-negative infections at the Medical University of South Carolina Medical Center, the use of aminoglycosides is considerably lower. For purposes of comparison, 
Table 1 Aminoglycoside usage for the years 1992 and 2006 through 2012 (defined daily doses/1,000 patient days)

\begin{tabular}{|c|c|c|c|c|c|c|c|c|c|c|}
\hline \multirow[t]{2}{*}{ Aminoglycoside } & \multicolumn{8}{|l|}{ Year } & \multicolumn{2}{|l|}{ \% Change } \\
\hline & 1992 & 2006 & 2007 & 2008 & 2009 & 2010 & 2011 & 2012 & $\begin{array}{l}1992 \text { versus } \\
2012\end{array}$ & $\begin{array}{l}2006 \text { versus } \\
2012\end{array}$ \\
\hline Amikacin & 41.2 & 3.4 & 5.0 & 4.9 & 11.6 & 4.6 & 10.7 & 4.7 & -88.5 & 39.2 \\
\hline Gentamicin & 46.5 & 16.6 & 14.2 & 24.6 & 21.4 & 20.7 & 23.1 & 22.9 & -50.5 & 38.3 \\
\hline Tobramycin & 32.3 & 98.8 & 93.1 & 133.1 & 126.0 & 121.1 & 130.6 & 140.0 & 333.0 & 41.7 \\
\hline Total & 120.0 & 118.8 & 112.2 & 162.6 & 159.0 & 146.4 & 164.3 & 167.7 & 39.7 & 41.2 \\
\hline$P^{\dagger}$ & - & - & - & - & - & - & - & - & 0.528 & 0.135 \\
\hline
\end{tabular}

†tudent's $t$ test; absolute change in DDD/1,000 PD

Table 2 Susceptibility to Aminoglycosides Over Time (\% susceptible)

\begin{tabular}{|c|c|c|c|c|c|c|c|c|c|}
\hline Aminoglycoside & Year & 1992 & 2006 & 2008 & 2009 & 2010 & 2011 & 2012 & $\boldsymbol{P}^{\dagger}$ \\
\hline \multicolumn{10}{|c|}{ Pseudomonas aeruginosa } \\
\hline & $n^{\mathrm{a}}$ & 306 & 379 & 197 & 235 & 126 & 194 & 180 & \\
\hline Amikacin & & 89 & 86 & 86 & 88 & 90 & 89 & 84 & 0.382 \\
\hline Gentamicin & & 71 & 70 & 81 & 85 & 85 & 87 & 80 & 0.439 \\
\hline Tobramycin & & 97 & 91 & 87 & 90 & 91 & 94 & 90 & 0.777 \\
\hline \multicolumn{10}{|l|}{ Escherichia coli } \\
\hline & $n^{\mathrm{a}}$ & 225 & 190 & 161 & 183 & 172 & 161 & 184 & \\
\hline Amikacin & & 100 & 97 & 97 & 98 & 98 & 99 & 98 & 0.617 \\
\hline Gentamicin & & 92 & 86 & 85 & 84 & 88 & 90 & 89 & 0.630 \\
\hline Tobramycin & & 98 & 87 & 82 & 83 & 87 & 87 & 89 & 0.661 \\
\hline \multicolumn{10}{|c|}{ Klebsiella pneumoniae } \\
\hline & $n^{\mathrm{a}}$ & 166 & 214 & 152 & 163 & 119 & 114 & 113 & \\
\hline Amikacin & & 99 & 82 & 93 & 94 & 96 & 98 & 98 & 0.597 \\
\hline Gentamicin & & 87 & 89 & 91 & 94 & 94 & 97 & 95 & 0.600 \\
\hline Tobramycin & & 87 & 79 & 88 & 92 & 92 & 96 & 92 & 0.866 \\
\hline
\end{tabular}

${ }^{\dagger}$ Chi-squared test; 1992 versus 2012

${ }^{a}$ Number tested

our 2012 annual usage of piperacillin/ tazobactam and meropenem were 228.5 and 595.4 DDD/1,000 PD, respectively (with DDDs defined as 1.5 and 20.25 grams, respectively) versus $120 \mathrm{DDD} / 1,000 \mathrm{PD}$ for all aminoglycosides combined.
Susceptibility of $P$. aeruginosa, E. coli and $K$. pneumoniae to these aminoglycosides did not change significantly over time either in the last few years of observation or compared to 1992 . While it has been suggested that there may be an increased interest and, therefore, use of 


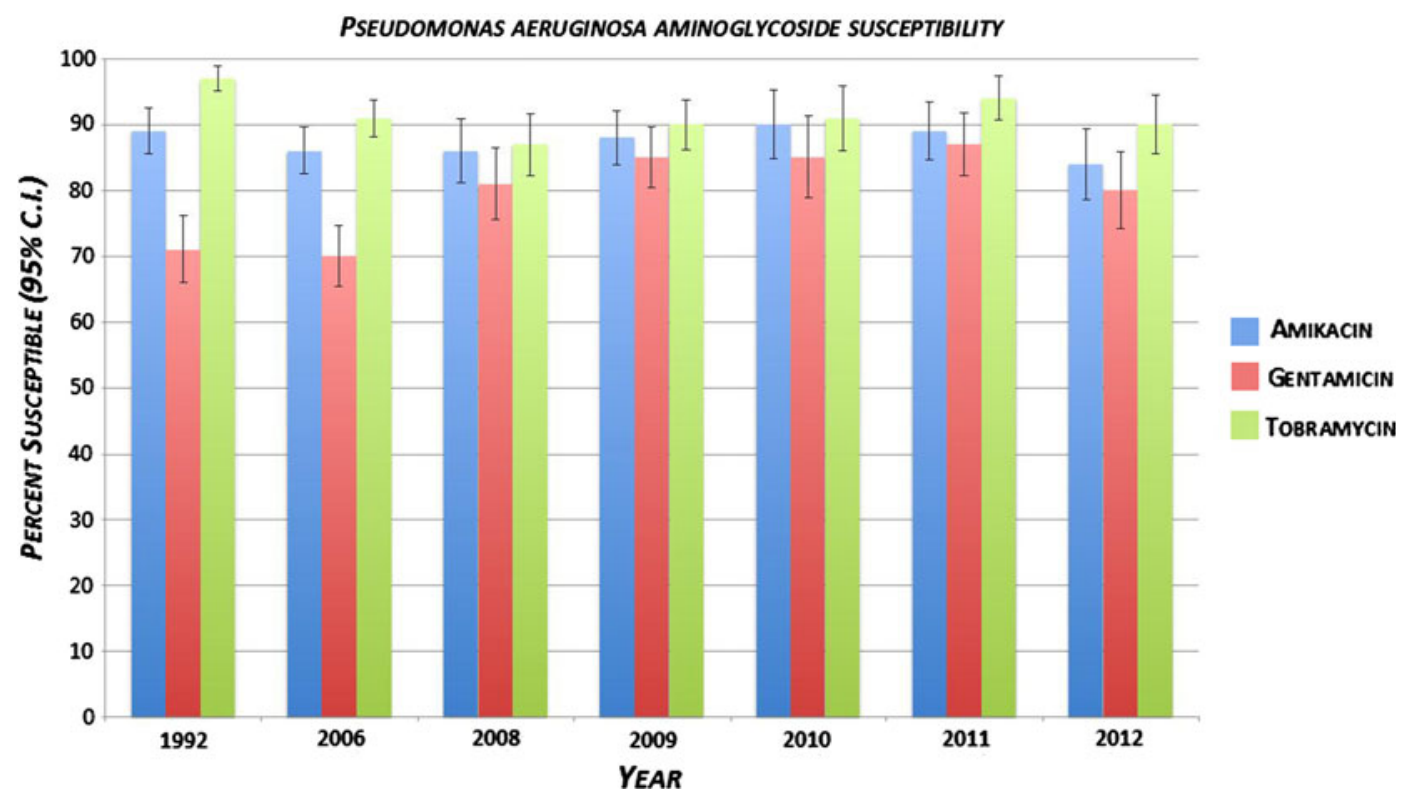

Fig. 1 Susceptibility of Pseudomonas aeruginosa over time [\% susceptible with $95 \%$ confidence interval (CI)]

aminoglycoside due to the emergence of widespread resistance of Enterobacteriaceae to betalactams mediated by ESBLs and carbapenemaseproducing Enterobacteriaceae [10-12], neither our observations nor those stemming from analyses using a collection of academic medical centers' data support that theory [1, 2]. In fact, the latter two studies revealed diminishing use [2]. In the more recent of these two reports, reflecting use in 29 hospitals, an overall decline of $41 \%$ was reported from 2002 to 2009 [2]. Resistance to aminoglycoside antibiotics occurs through a number of mechanisms including enzymatic modification, decreased cellular penetration, active efflux and target site alterations with the former being most common [15]. On that basis, it is reasonable to consider aminoglycoside therapy for infections involving Gram-negative pathogens suspected of producing newer ESBLs or carbapenemases. However, the observation that such bacteria often carry resistance determinants to other antibiotic classes, including aminoglycosides, fluoroquinolones and folic acid inhibitors, may undermine that line of thinking [7-9]. The fact that other broadspectrum antibiotic exposure may represent a risk factor for acquisition and infection by such organisms only exacerbates the challenge of identifying suitable therapy [16].

The positive aspect of our findings is perhaps that susceptibility of these key Gram-negative pathogens seems to be stable, at least at our institution. This may well be due to low levels of use in comparison with other Gram-negative agents. In fact, tobramycin remains the most active of our routinely tested antibiotics against $P$. aeruginosa while the vast majority of $E$. coli and $K$. pneumoniae are susceptible to amikacin. Thus, the aminoglycosides merit consideration in selecting antibiotic therapy for otherwise resistant Gram-negative pathogens. With our current level of understanding regarding proper aminoglycoside dosing, based upon pharmacodynamics characteristics [12], aminoglycosides represent potentially effective and relatively safe antibiotics. At the same time, it must be noted that a 2009 publication, 
reporting susceptibility data for a variety of bacteria including our organisms of interest collected and tested from 1999 through 2008, noted increasing aminoglycoside resistance [17]. That study collected isolates associated with serious infections from hospitals across the United States [17]. While levels of aminoglycoside use cannot be ascertained, that report emphasizes the importance of each hospital determining its own circumstances with regard to aminoglycoside susceptibility patterns [17].

The current study is not without limitations. As this is a single-center analysis, our results cannot be extrapolated to other hospitals or healthcare settings. We limited our investigation to $P$. aeruginosa, $E$. coli and $K$. pneumoniae as they are all common causes of healthcare associated infections and are often multidrug resistant [18]. Obviously, a number of other Gram-negative and Gram-positive pathogens are also problematic, multidrug resistant causes of healthcare associated infections and were not considered here. Because we used hospital antibiogram data, there could be an influence of including susceptibilities from both infecting and colonizing organisms on the values, as opposed to only considering organisms associated with documented infections. Further, the exclusion of duplicate isolates in an antibiogram constructed in compliance with CLSI guidance would theoretically ignore what might be more resistant samples thus skewing susceptibility proportion in a favorable way. We did not assess the influence of aminoglycoside "mix" (use of which agent predominated at various times) on resistance trends. Reports from the 1980s indicated that predominant use of amikacin at individual institutions resulted in improved gentamicin and/or tobramycin susceptibility among Gram- negative pathogens without a sacrifice in amikacin susceptibility $[19,20]$. Whether the changes in susceptibility we did observe between 1992 and 2012 in our pathogens of interest, none of which were statistically significant, were related to the change from predominant amikacin-gentamicin use to predominant tobramycin use is unknown. Further, whether these non-statistically significant changes were also clinically insignificant is a matter for consideration.

\section{CONCLUSION}

Low levels of aminoglycoside use, accompanied by stable susceptibility patterns in key Gramnegative pathogens, make these agents viable for treatment of serious infections for which other antibiotics may no longer be suitable.

\section{ACKNOWLEDGMENTS}

Dr. John Bosso is the guarantor for this article, and takes responsibility for the integrity of the work as a whole. No funding or sponsorship was received for this study or publication of this article.

Conflict of interest. John Bosso, Martha L. Haines, and Juanmanuel Gomez declare no conflict of interest.

Compliance with ethical guidelines. The study was approved by the Medical University of South Carolina Medical Center Institutional Review Board. This article does not contain any studies with human or animal subjects performed by any of the authors.

Open Access. This article is distributed under the terms of the Creative Commons Attribution Noncommercial License which 
permits any noncommercial use, distribution, and reproduction in any medium, provided the original author(s) and the source are credited.

\section{REFERENCES}

1. Pakyz AL, MacDougall C, Oinonen M, Polk RE. Trends in antibacterial use in US academic health centers: 2002-2006. Arch Intern Med. 2008; 168:2254-60.

2. Ababneh M, Harpe S, Oinonen M, Polk RE. Trends in aminoglycoside use and gentamicin-resistant Gram-negative clinical isolates in US academic medical centers: implications for antimicrobial stewardship. Infect Control Hosp Epidemiol. 2012;33:594-601.

3. Gerding DN, Larson TA, Hughes RA, Weiler M, Shanholtzer C, Peterson LR. Aminoglycoside resistance and aminoglycoside usage: ten years of experience in one hospital. Antimicrob Agents Chemother. 1991;35:1284-90.

4. Weinstein RA, Nathan C, Gruensfelder R, Kabins SA. Endemic aminoglycoside resistance in Gramnegative bacilli: epidemiology and mechanisms. J Infect Dis. 1980;141:338-45.

5. Jacoby GA, Munoz-Price LS. The new $\beta$-lactamases. N Engl J Med. 2005;352:280-91.

6. Savard P, Carroll KC, Wilson LE, Perl TM. The challenges of carbapenemase-producing Enterobacteriaceae and infection prevention: protecting patients in the chaos. Infect Control Hosp Epidemiol. 2013;34:730-9.

7. Kallen AJ, Srinivasan A. Current epidemiology of multidrug-resistant Gram-negative bacilli in the United States. Infect Control Hosp Epidemiol. 2010;31(S1):S51-4.

8. Schwaber MJ, Navon-Venezia S, Schwartz D, Carmeli Y. High levels of antimicrobial coresistance among extended-spectrum- $\beta$ lactamase-producing Enterobacteriaceae. Antimicrob Agents Chemother. 2005;49(5):2137-9.

9. Colodner R, Samra Z, Keller N, et al. First national surveillance of susceptibility of extended-spectrum $\beta$-lactamase-producing Escherichia coli and Klebsiella spp. to antimicrobials in Israel. Diagn Microbiol Infect Dis. 2007;57(2):201-5.

10. Leibovici L, Vidal L, Paul M. Aminoglycoside drugs in clinical practice: an evidence-based approach. J Antimicrob Chemother. 2009;63:246-51.
11. Mueller EW, Boucher BA. The use of extendedinterval aminoglycoside dosing strategies for the treatment of moderate-to-severe infections encountered in critically ill surgical patients. Surg Infect. 2009;10:563-70.

12. Drusano GL, Ambrose P, Louie A. Optimization of aminoglycoside therapy. Antimicrob Agents Chemother. 2011;55:2528-31.

13. Clinical and Laboratory Standards Institute (CLSI). Analysis and presentation of cumulative antimicrobial susceptibility test data. 3rd ed. Approved guideline M39-A3. Wayne, PA: CLSI; 2009.

14. Juretschko S, LaBombardi VJ, Lerner SA, Sreckenberger PC. Accuracies of $\beta$-lactam susceptibility test results for Pseudomonas aeruginosa with four automated systems (BD Phoenix, MicroScan WalkAway, Vitek and Viteck 2). J Clin Microbiol. 2007;45:1339-42.

15. Jana S, Deb JK. Molecular understanding of aminoglycoside action and resistance. Appl Microbiol Biotechnol. 2006;70:140-50.

16. Wener KM, Schechner V, Gold HS, Wright SB, Carmeli Y. Treatment with fluoroquinolones or with $\beta$-lactam- $\beta$-lactamase inhibitor combinations is a risk factor for isolation of extended-spectrum- $\beta$ lactamase-producing Klebsiella species in hospitalized patients. Antimicrob Agents Chemother. 2010;43:2010-6.

17. Rhomberg PR, Jones RN. Summary trends for the meropenem yearly susceptibility test information collection program: a 10-year experience in the United States (1999-2008). Diagn Microbiol Infect Dis. 2009; 65:414-26.

18. Hidron AI, Edwards JR, Patel J, et al. Antimicrobialresistant pathogens associated with healthcareassociated infections: annual summary of data reported to the National Healthcare Safety Network at the Centers for Disease Control and Prevention, 2006-2007. Infect Control Hosp Epidemiol. 2008;29:996-1011.

19. Gerding DN, Larson TA. Aminoglycoside resistance in gram-negative bacilli during increased amikacin use. Comparison of experience in 14 United States hospitals with experience in the Minneapolis Veterans Administration Medical Center. Am J Med. 1985;79:1-7.

20. Betts RF, Valenti WM, Chapman SW, et al. Five-year surveillance of aminoglycoside usage in a university hospital. Ann Intern Med. 1984;100:219-22. 